STRUCTURAL BIOLOGY COMMUNICATIONS

ISSN 2053-230X

\section{Comparative structure analysis of the ETSi domain of ERG3 and its complex with the E74 promoter DNA sequence. Corrigendum}

\author{
Ruby Sharma, Shanti P. Gangwar and Ajay K. Saxena*
}

Structural Biology Laboratory, School of Life Sciences, Jawaharlal Nehru University, New Delhi 110 067, India. *Correspondence e-mail: ajaysaxena@mail.jnu.ac.in

The article by Sharma et al. [(2018), Acta Cryst. F74, 656-663] is corrected.

Edited by M. J. Romao, Universidade Nova de Lisboa, Portugal

Keywords: prostate cancer; ETS transcription factor; ERG3; E74 promoter DNA; corrigendum.

An incorrect SDS-PAGE image was added by mistake to Fig. 1 of the article by Sharma et al. (2018). The correct version is published here.
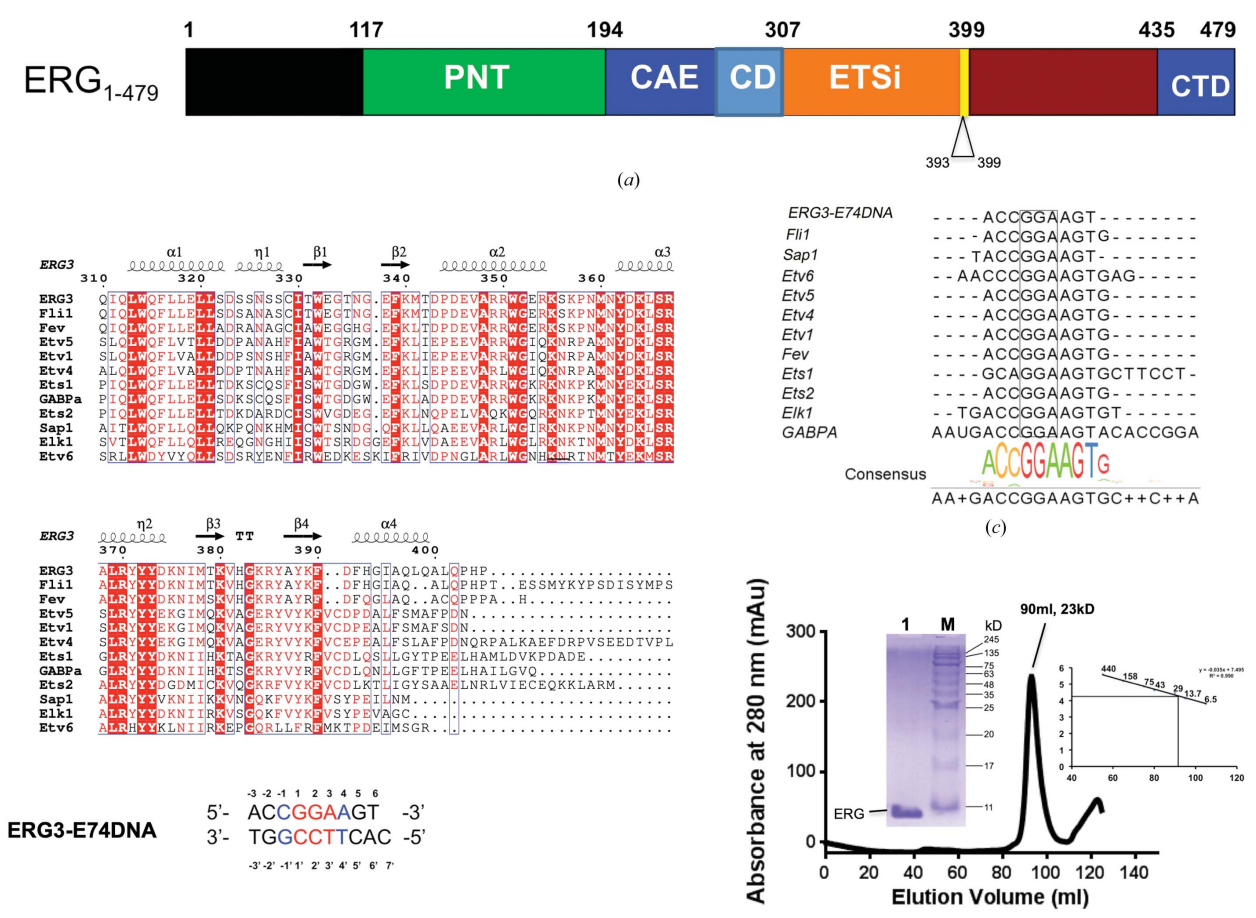

(d)

Figure 1

(a) Schematic view of the various domains of ERG3 proteins. The ETSi construct used in the current study contains residues 307-392 of the ETS domain and residues 393-399 of the adjacent CID domain of ERG3 (shown in yellow). (b) Sequence alignment of the ETSi domain of ERG3 with sequences from 11 other ETS class 1 protein-DNA complexes of known structure: Fli1 (PDB entry 5e8i), Fev (PDB entry 3zp5), Etv5 (PDB entry 4uno), Etv1 (PDB entry 4bnc), Etv4 (PDB entry 4uuv), Ets1 (PDB entry 3mfk), GABP $\alpha$ (PDB entry 1awc), Ets2 (PDB entry 4bqa), Sap1 (PDB entry 1bc7), Elk1 (PDB entry 1dux) and Etv6 (PDB entry 4mhg). The MultiAlin (Corpet, 1988) and ESPript 3.0 programs (Robert \& Gouet, 2014) were used for multiple sequence alignment and structure prediction. The secondary structures of the ETSi domain are shown above the sequence alignment, with $\alpha$-helices as spirals and $\beta$-strands as arrows. Highly conserved residues are shaded red and less conserved residues are shown as red and blue letters. The lower panel shows the DNA, sequence used in the current study. (c) The DNA sequence of the ETSi-DNA $_{9}$ complex is aligned with the DNA sequences of 11 ETS class 1 protein-DNA complexes. Except for the DNA sequences from the ETS1-DNA and ETV6-DNA complexes, the nine base pairs of other ETS class 1 protein-DNA complexes are similar to the DNA, sequence. $(d)$ Size-exclusion chromatography and SDS-PAGE analysis of the purified ETSi domain. The ETSi domain eluted as a dimer from the size-exclusion column and shows a single band on SDS-PAGE. 


\section{addenda and errata}

\section{References}

Corpet, F. (1988). Nucleic Acids Res. 16, 10881-10890.

Robert, X. \& Gouet, P. (2014). Nucleic Acids Res. 42, W320-
W324.

Sharma, R., Gangwar, S. P. \& Saxena, A. K. (2018). Acta Cryst. F74, 656-663. 